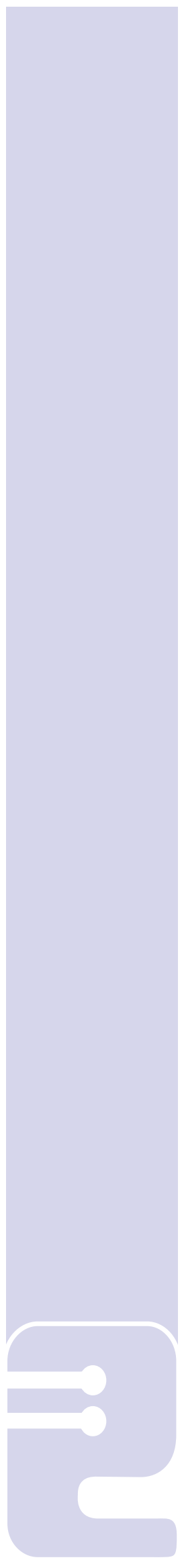

\title{
Caracterización del tráfico en redes móviles ad-hoc basada en series de tiempo y redes neuronales recurrentes
}

\author{
Gabriel A. Puerta', Pablo U. Fonseca²
}

Recibido: Agosto 20 de 2014 Aprobado: Diciembre 08 de 2014

\begin{abstract}
Resumen:
El objetivo de este artículo es implementar una metodología de análisis y estimación del comportamiento del tráfico de paquetes en redes móviles Ad-hoc. Se construyeron procesos de estimación de tráfico en redes móviles con base en la implementación de redes neuronales recurrentes y series de tiempo; para ello se trabajaron 50 muestras de diferentes escenarios de caracterización de variables de entorno de forma aleatoria, simulados en NS-2 (Network Simulator Two), luego estas trazas fueron procesadas con la ayuda de herramientas como AWK y Perl. Se consiguieron históricos de 1300 y 3000 eventos para su posterior validación frente al estimador. Los estimadores y las caracterizaciones de tráfico implementadas para las redes neuronales, estan basadas en la regla Back Propagation y modelos de regresión lineal como ARIMA, SARIMA y FARIMA, para las series de tiempo.
\end{abstract}

Palabras clave: Modelos autoregresivos, Redes neuronales, Teletráfico, Estimación.

\section{Abstract:}

The goal of this paper is mainly to construct a methodology to analyze and estimate the behavior of traffic in mobile ad-hoc networks. Estimation traffic processes are based on recurrent neural networks and time series; there were 50 samples drawn from the scenarios, generating random simulation in NS-2 and then treated through Perl and Awk. We get historical values of 1300 and 3000 events that served as reference data for validation. The estimation process was based on a neuronal network of the type back propagation and also on linear regression models als ARIMA, SARIMA y FARIMA for time series.

Keywords: Auto regresive models, Neuronal networks, Teletraffic, Estimation.

\footnotetext{
${ }^{1}$ Licenciado en Electrónica, máster en Ciencias de la Información y las Comunicaciones, docente del programa de Tecnología en Redes de Computadores y Seguridad Informática, UNIMINUTO - Sede Principal.

2 Ingeniero Eléctrico, especialista en Redes de Alta Velocidad y Distribuidas, docente del programa de Tecnología en Redes de Computadores y Seguridad Informática, UNIMINUTO - Sede Principal.
} 


\section{INTRODUCCIÓN}

La teoría de tráfico de paquetes se enmarca en la generación y aplicación de modelos matemáticos que describan la relación que existe entre la capacidad de una red de comunicaciones, la caracterización de los usuarios y su demanda de servicios [1], [2]. Estas demandas constituyen un comportamiento de orden estadístico, razón por la cual es factible partir de procesos estocásticos para caracterizar o expresar los distintos modelos de tráfico que puedan describir o estimar estos comportamientos [3], [4].

Existen diferentes modelos de tráfico que de acuerdo con el uso que se les logre dar a sus herramientas, pueden convertirse en un punto de partida para el diseño de mecanismos de control y administración en redes en un variado número de aspectos tales como: control de admisión, control de acceso a la red, control de recursos, control de flujos, control de memoria, enrutamiento dinámico y control de congestión entre otros.

Para el caso de las redes telefónicas, la relación que hay entre desempeño y tráfico se describe por la fórmula de Erlang $B$, la cual entrega la probabilidad de que una llamada no pueda acceder a la red o sea rechazada [1], para $N$ circuitos sobre los que hay una intensidad de tráfico $P$ ro, definida como la tasa de arribo de llamadas, por la duración promedio de estas.

$$
P_{B}=\frac{\frac{\rho^{N}}{N !}}{\sum_{n=0}^{N} \frac{\rho^{n}}{n !}}
$$

En esta descripción se asume que los arribos de las llamadas obedecen a un proceso probabilístico estacionario de Poisson, haciendo que la probabilidad de bloqueo del sistema no dependa de algunos aspectos importantes en las redes como la distribución de tiempos entre llamadas [1]. En un proceso de Poisson los arribos se producen en intervalos exponencialmente distribuidos y a su vez estos son procesos independientes. De esta forma el número de arribos en cualquier intervalo de tiempo T está dado por:

$$
P[A(t+T)-A(t)]=n=\frac{(\lambda T)^{n}}{n !} e^{-\lambda T}
$$

El tiempo de espera entre un arribo y otro viene dado por:

$$
P[N=m+k \vee N>k]=
$$

$$
\begin{gathered}
m-1 \\
\forall k \geq 0 \\
p(1-p)
\end{gathered}
$$

Durante algunas décadas los procesos de Poisson con las ecuaciones enunciadas anteriormente, fueron usados con gran éxito en el control de redes telefónicas y en redes de datos en tecnologías como X25 [4]. Esto debido a que para ese entonces las redes presentaban uniformidad de servicios y un rango pequeño de capacidades en sus medios de transmisión.

\section{ALGUNAS TÉCNICAS DE ESTIMACIÓN DE TRÁFICO}

Las redes con mayores capacidades y servicios hicieron que la teoría de tráfico y colas pasara del análisis de flujos de conexión, al análisis de tráfico a través de paquetes de datos. Hasta el día de hoy se han aplicado un variado número de modelos para tratar de estimar el comportamiento del tráfico de una red, con sus respectivas técnicas y herramientas como pueden ser las series de tiempo [5], [6], [7], [8] o los modelos auto-regresivos, entre ellos:

ARIMA

ARMA

FARIMA

SARIMA

Estas técnicas se basan en obtener un histórico del comportamiento del tráfico, luego extraer las relaciones que existen entre los valores pasados para de esta forma tratar de estimar algunos valores futuros. La denominación de estos modelos viene dada por sus siglas en inglés:

Auto regressive AR,

Moving average MA

Integrated I

Fraccional $\mathrm{F}$

Seasonal S

Estos son los modelos AR (Auto regresivos) [9], los modelos MA (Media móvil), los ARMA (Auto regresivos con promedio móvil), los ARIMA (Auto regresivos integrados con media móvil), los FARIMA (Auto regresivo integrado de promedio móvil fraccional) y los SARIMA (Auto regresivo de media móvil estacionario) [9], [10], [11], [12], [13]. A continuación se desglosan algunas de las características de estos modelos.

Auto regresión: Para comenzar se considera un modelo AR (1) auto regresivo de orden uno. 


$$
y_{t} \varphi y_{t-1}+\varepsilon_{t}
$$

Donde la variable independiente está dada por el valor que toma en el instante anterior más un error interno o endógeno.

$$
\operatorname{siE}\left(\varepsilon_{t}\right)=0 \wedge E\left(\varepsilon_{t} * y_{t-1}\right)=0
$$

El error endógeno no solo estará dado en términos de una correlación serial, sino que también estará dado en términos de una variación exógena:

$$
\varepsilon_{t} \rho \varepsilon_{t}+\eta_{t}
$$

De esta forma la relación será:

$$
y_{t} \varphi y_{t-1}+\varepsilon_{t}
$$

Donde $y_{t-1}$ dependerá solo del error endógeno del valor anterior, al igual que $\varepsilon_{t}$.

Uno de los elementos más importantes en la ingeniería de teletráfico en los últimos años es el análisis de tráfico multifractal [5]; este tipo de tráfico es el resultado de la información generada por los usuarios al enviar y generar paquetes a los dispositivos de enrutamiento, los cuales se encargan de gestionar la entrega de la información en su destino [5], [14], [15]. Los dispositivos de enrutamiento presentan ciertas características de encolamiento tales como: Tiempos de servicio, tamaño de los buffers y tasas de arribo entre otros. El comportamiento del tráfico de estos dispositivos se modela bajo dos características: el tráfico no auto-similar y el tráfico auto-similar [16], en el primero caso están contenidos los modelos como: fuentes ON-OFF, procesos de Poisson, distribuciones Gaussianas y Moduladamente Markovianos; estos modelos presentan determinadas distribuciones de tiempos y paquetes.

El Modelo Wavelet Multifractal (MWM) es un modelo de tráfico [16], [17] que se basa en la captura de las principales características estadísticas del tráfico en redes con gran eficiencia computacional [5], [6], [18], la herramienta principal de análisis de este modelo es la transformada wavelet discreta, la cual representa a una señal real unidimensional $x t$ [19] construida en términos del cambio de desplazamiento y la versión dilatada de una función wavelet pasa bandas $\psi t$ [20]; también se considera el cambio de una función de escala pasa bajos $\varphi t$ para algunas funciones wavelet y de escala seleccionadas, las versiones dilatadas y desplazadas $\phi_{j, k} t:=2^{j / 2} \phi\left(2^{j} t-k\right)$ y su base ortogonal $0 j, k t:=2^{j / 2} 0\left(2^{j} t-k\right) \forall j, k \in Z$ así la señal puede ser representada [16], [17].

$$
\begin{gathered}
X t=\sum_{k} U_{J o k} o_{\cdot j o, k} t+\sum_{j=j o}^{\infty} \cdot \sum_{k} W j k \emptyset j k t \\
W_{j, k}:=\int X t \psi_{j, k} t d t
\end{gathered}
$$

Adicional a los modelos y técnicas antes nombradas, hace algún tiempo se viene trabajando la implementación de sistemas neuronales artificiales para la caracterización del tráfico de distintas redes bajo distintas condiciones, basados en los resultados que han dado las redes neuronales en la predicción de muchos procesos en la naturaleza y el mundo actual (economía, reconocimiento de patrones, etc.) [20], [21], [22].

Es por esta razón que este artículo se centró en la caracterización de un modelo de tráfico en redes móviles Ad-hoc, bajo direccionamiento Ipv4 e Ipv6 con fuentes de tráfico de video en tiempo real, aplicando redes neuronales, con el fin de estimar comportamientos futuros del tráfico característico.

\subsection{Redes Neuronales}

Para hablar de redes neuronales artificiales es coherente revisar a manera introductoria, el comportamiento de las redes neuronales biológicas [23].

En nuestro sistema nervioso existen unas células llamadas neuronas, que funcionan como una unidad simple de procesamiento que reciben estímulos eléctricos de otras neuronas a través de las dendritas [24], [25], [26]; este estimulo eléctrico recibido, al superar cierto umbral, logra que la neurona envíe una señal eléctrica a través de su axón [27], [28], a otras neuronas que se encuentren interconectadas a través de la sinapsis [29], [30].

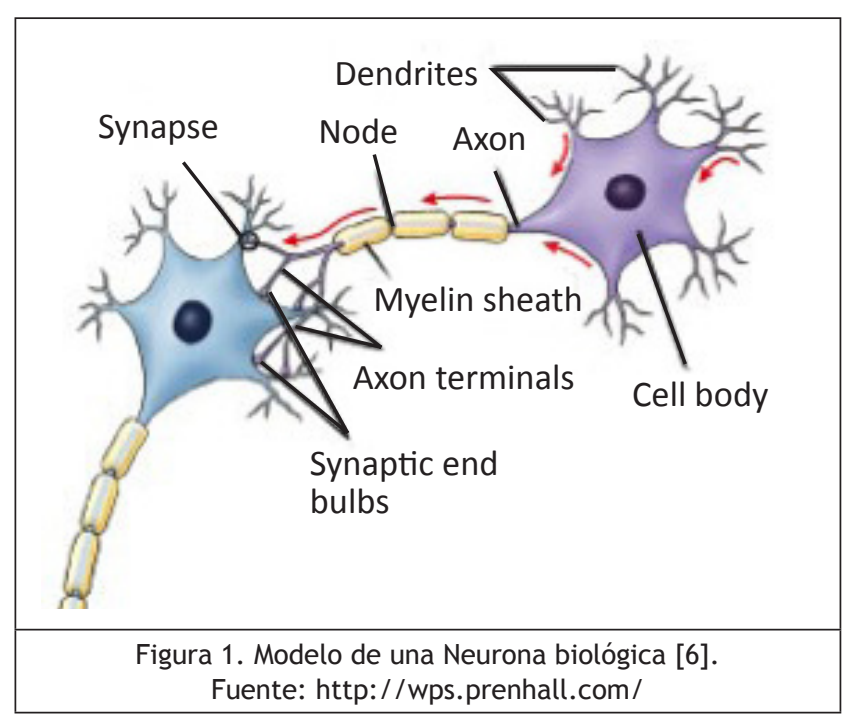


Basados en el funcionamiento biológico de las neuronas nacen las redes de neuronas artificiales.

\section{METODOLOGÍA}

Una vez diseñados los escenarios de simulación bajo redes móviles Ad-hoc en NS-2 (Network Simulator Two).

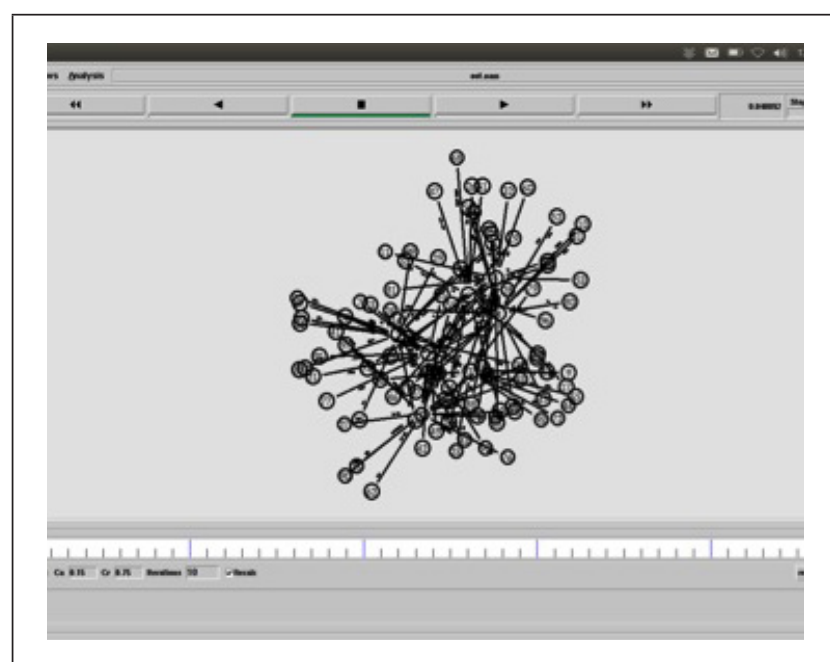

Figura 2. Topología escenario aleatorio. Fuente: Autores

Las trazas de captura de todos los eventos ocurridos en la simulación, son generadas por el simulador en archivos de texto plano; estos archivos son procesados a través de herramientas de procesamiento de texto Perl y Awk.

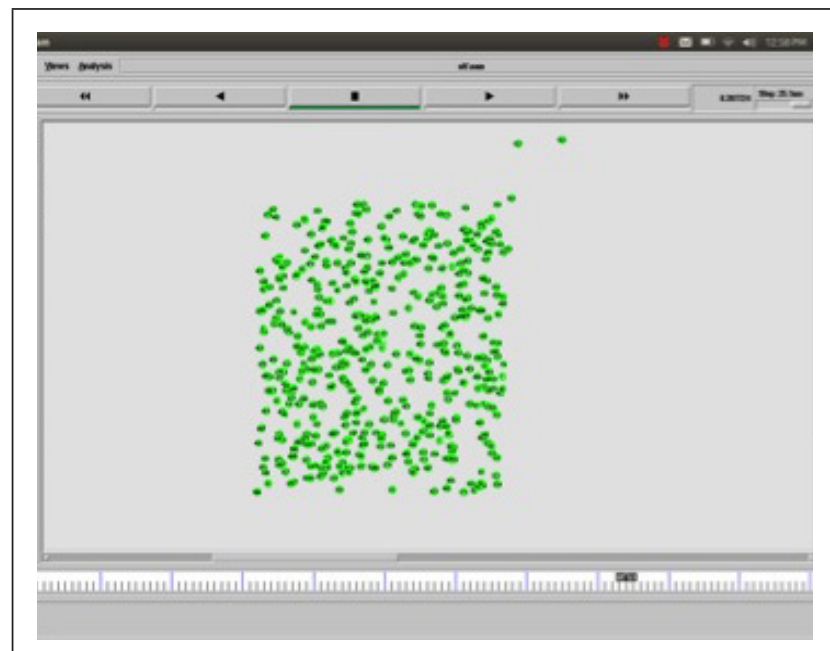

Figura 3. Topología escenario aleatorio con movilidad. Fuente: Autores

Se evalúan en promedio 50 muestras de 16000 eventos, después estas muestras o trazas se dividen eventos, el segundo intervalo contiene 3000 eventos. La traza que contiene 1300 eventos se dimensionó a la medición del "throughput" por valor promedio cada 100 eventos, obteniendo como resultado una traza final de 130 valores promedio del "throughput" de la red.

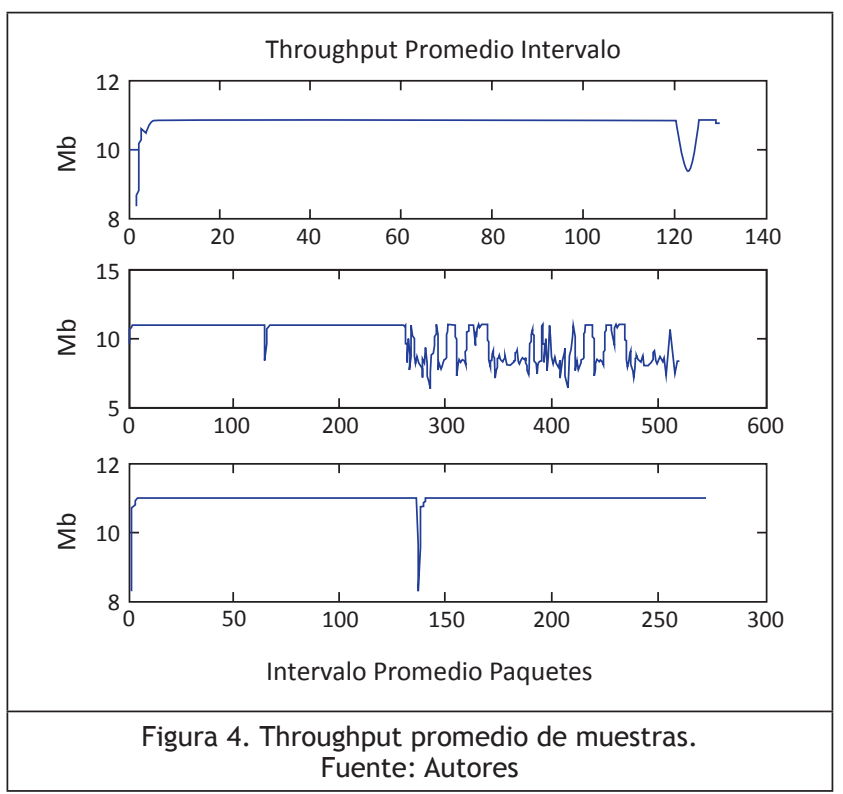

Después de haber obtenido los datos promedio por intervalo, se construyó una red neuronal de tipo Back Propagation, a la cual se le ingresaron como entradas los vectores de tiempo de cada intervalo y el valor promedio en Megabytes de transmisión de cada intervalo de las 50 muestras, para generar el aprendizaje de la red y ajustar su proceso de estimación.

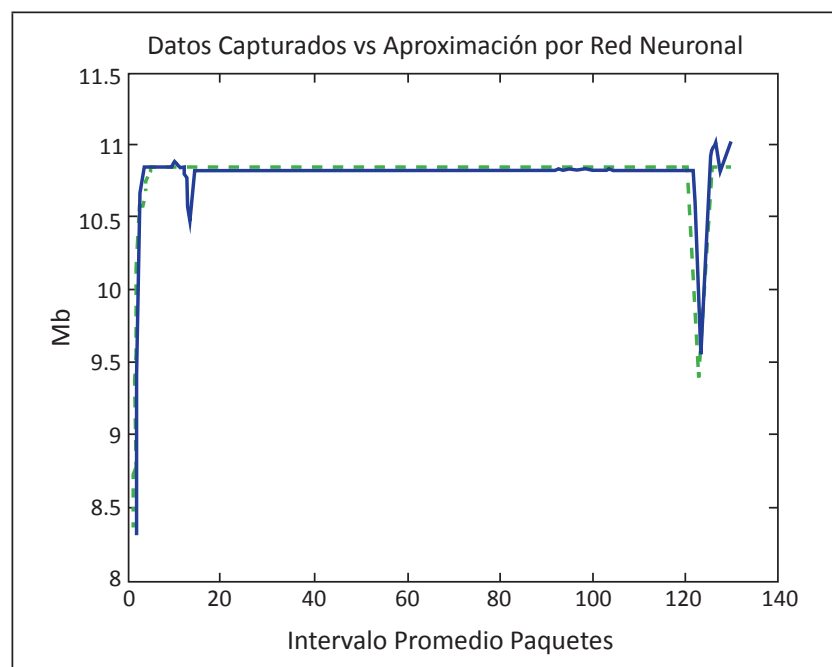

Figura 5. Throughput promedio Vs valor estimado red móvil Ad-hoc IPV6. Fuente: Autores 
Una vez entrenada la red, se realizó la simulación de la misma para lograr caracterizar la salida de la red neuronal y el comportamiento del tráfico generado por los escenarios de simulación, obteniendo los siguientes resultados.

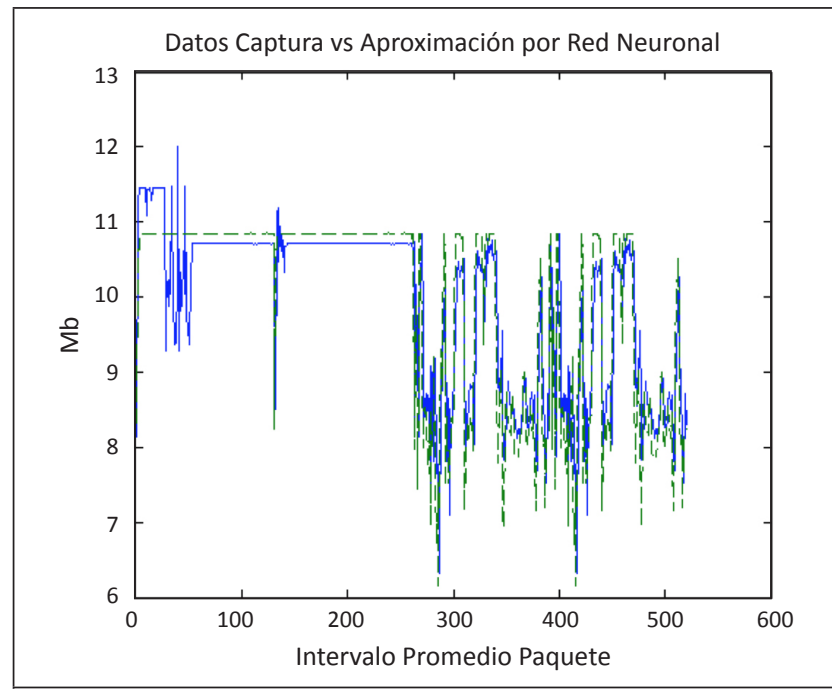

Figura 6. Throughput promedio Vs valor estimado red móvil Ad-hoc IPV4. Fuente: Autores

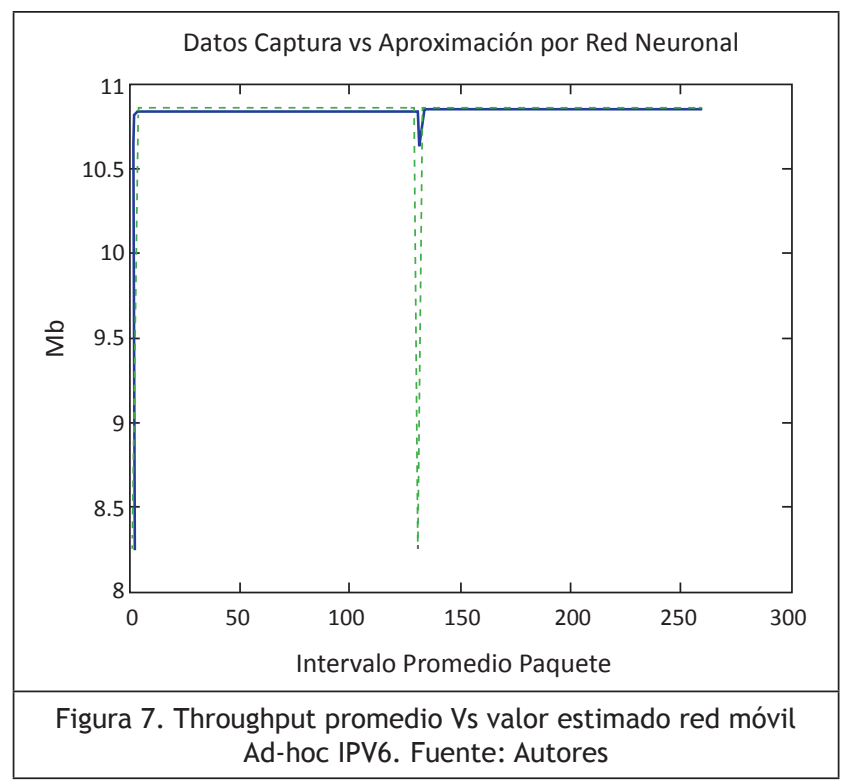

\section{CONCLUSIONES}

Las series de tiempo son una herramienta util para reconstruir el comportamiento del tráfico de una red, pero asociado a la tenencia de un historial relativamente extenso, ya que se pierde la eficiencia de los pronósticos para eventos con orden de regresión menor a tres (3).

Las redes neuronales recurrentes se adaptan fácilmente al comportamiento del tráfico en redes móviles, convirtiéndose en una herramienta para el análisis de correlación de eventos de una red.

Las redes móviles Ad-hoc representan un desafío en el avance de las redes, puesto que aún se encuentran en fase de mejoramiento especialmente en cuanto al comportamiento de los nodos y su consumo energético.

El dinamismo de las redes y los cambios no periódicos de sus topologías constituyen una implicación directa en el desempeño de las variables de una red, tales como throughoutput, jitter, pérdidas y retardos.

\section{REFERENCIAS BIBLIOGRÁFICAS}

1. G. Alzate, M.; Peña, N.; Modelos de tráfico en redes modernas de comunicaciones, Revista INGENIERIA, Universidad Distrital, 2004, Vol. 9.

2. Alzate, M.; Introducción al Tráfico autosimilar en Redes de Comunicaciones, Revista INGENIERIA, Universidad Distrital, 2001, Vol. 6.

3. Aguirre, E., Puerta, A. Comparación del modelo wavelet multifractal entre una red Ethernet y una red Manet, Revista TECNURA Universidad Distrital 2011, Vol. 15 No. 29.

4. Alzate, M. End-to-end Available Bandwidth Estimation in IEEE 802.11b Ad Hoc Networks. Tesis Doctoral en Ingeniería Electrónica, Universidad de los Andes, diciembre de 2008

5. Jung-Hua Wang; Jia-Yann Leu, "Stock market trend prediction using ARIMA-based neural networks," Neural Networks, 1996., IEEE International Conference on, vol.4, 3-6 Jun 1996.

6. Seng-Cho Timothy Chou; Chau-Chen Yang; ChiHuang Chan; Feipei Lai, "A rule-based neural stock trading decision support system," Computational Intelligence for Financial Engineering, 1996., Proceedings of the IEEE/IAFE 1996 Conference on, vol., no., pp.148,154, 24-26 Mar 1996.

7. Amorim Neto, M.C.; Tavares, G.; Alves, V. M O; Cavalcanti, G. D C; Tsang Ing Ren, "Improving financial time series prediction using exogenous series and neural networks committees," Neural Networks (IJCNN), The 2010 International Joint Conference on, vol., no., pp.1,8, 18-23 July 2010.

8. Silva, G.A.; Culp, B., "High Throughput Algorithms for Mapping the Topology of Neuronal and Glial Networks," Neural Engineering, 2005. 
Conference Proceedings. 2nd International IEEE EMBS Conference on , vol., no., pp.344,347, 1619 March 2005.

9. Lin Chen; Weihua Luo; Yong Deng; Zhen Wang; Zeng, Shaoqun, "Characterizing the Complexity of Spontaneous Electrical Signals in Cultured Neuronal Networks Using Approximate Entropy," Information Technology in Biomedicine, IEEE Transactions on, vol.13, no.3, pp.405,410, May 2009.

10. Esposti, F.; Signorini, M.G.; Cerutti, S., "Neuronal networks and Self-Organized Criticality: The rising of long-term memory in neuronal ensembles," Neural Engineering, 2009. NER '09. 4th International IEEE/EMBS Conference on, vol., no., pp.538,541, April 29 2009-May 22009.

11. Stegenga, J.; Le Feber, J.; Marani, E.; Rutten, W. L C, "Analysis of Cultured Neuronal Networks Using Intraburst Firing Characteristics," Biomedical Engineering, IEEE Transactions on, vol. 55, no.4, pp.1382,1390, April 2008

12. Maffezzoli, A.; Signorini, M.G.; Gullo, F.; Wanke, E., "Modeling spiking activity of in vitro neuronal networks through non linear methods," Engineering in Medicine and Biology Society, 2008. EMBS 2008. 30th Annual International Conference of the IEEE, vol., no., pp.42,45, 2025 Aug. 2008

13. Tarraf, A.A.; Habib, I.W.; Saadawi, T.N.; “A novel neural network traffic enforcement mechanism for ATM networks," Selected Areas in Communications, IEEE Journal on, vol. 12, no.6, pp.1088-1096, Aug 1994

14. Razavi, S.N.; Fathy, M.; "Qualitative traffic analysis using image processing and time-delayed neural network," Intelligent Transportation Systems, 2002. Proceedings. The IEEE 5th International Conference.

15. Yuh-Horng Wen; Tsu-Tian Lee; , "Fuzzy data mining and grey recurrent neural network forecasting for traffic information systems," Information Reuse and Integration, Conf, 2005. IRI -2005 IEEE International Conference on., vol., no., pp. 356-361, 15-17 Aug. 2005

16. Runyuan Sun; Bo Yang; Lizhi Peng; Zhenxiang Chen; Lei Zhang; Shan Jing; "Traffic classification using probabilistic neural networks," Natural
Computation (ICNC), 2010 Sixth International Conference on, vol. 4.

17. Mehrvar, H.R.; Le-Ngoc, T.; Huang, J.; "Performance evaluation of bursty traffic using neural networks," Electrical and Computer Engineering, 1996. Canadian Conference on, vol.2, no., pp. 955-958 vol. 2

18. Shu-Yan Chen; Gao-Feng Qu; Xing-He Wang; Huai-Zhong Zhang;, "Traffic flow forecasting based on grey neural network model," Machine Learning and Cybernetics, 2003 International Conference on, vol. 2.

19. Gavade, J. D.; Kharat, P.K.; "Neural Network based approach for MPEG video traffic prediction," Advances in Recent Technologies in Communication and Computing (ARTCom 2011), 3rd International Conference 2011.

20. Ohe, I.; Kawashima, H.; Kojima, M.; Kaneko, Y.; "A method for automatic detection of traffic incidents using neural networks," Vehicle Navigation and Information Systems Conference, 1995. Proceedings. In conjunction with the Pacific Rim TransTech Conference. 6th International VNIS. 'A Ride into the Future', vol., no., pp.231-235, 30 Jul-2Aug 1995.

21. Xiaojun Shen; Chen, J.;, "Study on Prediction of Traffic Congestion Based on LVQ Neural Network," Measuring Technology and Mechatronics Automation, 2009. ICMTMA '09. International Conference on , vol.3, no., pp.318-321, 11-12 April 2009.

22. Yuming Mao; Shiying Shi; " "Research on Method of the Subsection Learning of Double-Layers $B P$ Neural Network in Prediction of Traffic Volume," Measuring Technology and Mechatronics Automation, 2009. ICMTMA '09. International Conference on, vol. 3

23. Qin Liyan; Shao Chunfu; "Macro Prediction Model of Road Traffic Accident Based on Neural Network and Genetic Algorithm," Intelligent Computation Technology and Automation, 2009. ICICTA '09. Second International Conference on, vol.1

24. Cong Wang; Xiaoxia Zhang; Han Yan; Linlin Zheng;, "An Internet Traffic Forecasting Model Adopting Radical Based on Function Neural Network Optimized by Genetic Algorithm," Knowledge Discovery and Data Mining, 2008. WKDD 2008. 
First International Workshop on, vol., no., pp.367-370, 23-24 Jan. 2008.

25. Xiaodong Zang;, "The short-term traffic volume forecasting for urban interchange based on RBF Artificial Neural Networks," Mechatronics and Automation, 2009. ICMA 2009. International Conference on, vol., no., pp.2607-2611, 9-12 Aug. 2009

26. Tarraf, A.A.; Habib, I.W.; Saadawi, T.N.; “A novel neural network traffic enforcement mechanism for ATM networks," Communications, 1994. ICC '94, SUPERCOMM/ICC '94, Conference Record, 'Serving Humanity Through Communications.' IEEE International Conference

27. Gang Tong; Chunling Fan; Fengying Cui; Xiangzhong Meng; "Fuzzy Neural Network Model Applied in the Traffic Flow Prediction," Information Acquisition, 2006 IEEE International Conference on, vol., no., pp.1229-1233, 20-23 Aug. 2006.
28. Yao, X.; Fischer, M.; Brown, G.;, "Neural network ensembles and their application to traffic flow prediction in telecommunications networks," Neural Networks, 2001. Proceedings. IJCNN '01. International Joint Conference on, vol.1, no., pp. 693-698 vol.1, 2001

29. Chen Di; Feng Hai-Hang; Lin Qing-jia; Chen Chunxiao;, "Multi-scale Internet Traffic Prediction Using Wavelet Neural Network Combined Model", Communications and Networking in China, 2006. ChinaCom '06. First International Conference on, vol., no., pp.1-5, 25-27 Oct. 2006

30. Lu Baichuan; Huang Meiling; "Traffic Flow Prediction Based on Wavelet Analysis, Genetic Algorithm and Artificial Neural Network," Information Engineering and Computer Science, 2009. ICIECS 2009. International Conference on, vol., no., pp.1-4, 19-20 Dec. 2009. 